Revista de Matemática: Teoría y Aplicaciones 2000 7(1-2) : 91-106

CIMPA - UCR ISSN: 1409-2433

\title{
GRAFOS DESTINADOS AL ANÁLISIS DE DATOS MULTIVARIANTE CONDICIONADO POR SU ESTRUCTURA*
}

\author{
Miguel Angel García Montoya ${ }^{\dagger}-$ Amaya Zárraga Castro ${ }^{\ddagger}$ \\ Belén Castro IÑigo $§$
}

Recibido: 23 Mayo 2000

\begin{abstract}
Resumen
En este artículo se muestran dos nuevos métodos para la construcción de grafos que pueden utilizarse en combinación con las técnicas descriptivas de Análisis de Datos Multivariante: grafo basado en la distancia ji-cuadrado y grafo basado en las componentes fuertemente conectadas.

Además se presentan sendos ejemplos correspondientes a cada uno de los grafos, tomando como datos la matriz intermedia de las Tablas Input-Output.
\end{abstract}

Palabras clave: Análisis Factorial de Correspondencias condicionado por un grafo, Tablas Input-Output, Teoría de Grafos, Distancia ji-cuadrado, Componentes Fuertemente Conectadas.

\begin{abstract}
We present two new methods for the construction of graphs that can be used with descriptive techniques of Multivariate Data Analysis: a graph based in the chi-square distance and a graph based in strongly-connected components.

We also present exemples corresponding to the graphs, using data from an intermediate Input-Output Matrix.
\end{abstract}

Keywords: Correspondence Factor Analysis conditioned to a graph, Input-Output Tables, Graph Theory, Chi-square dictance, Strongly connected components.

Mathematics Subject Classification: 65H25, 05C50

\footnotetext{
*Este trabajo está financiado por el proyecto UPV 036.351-HA062/99

${ }^{\dagger}$ Instituto de Investigación Operativa y Estadística, Facultad de Ciencias Económicas y Empresariales, Universidad del País Vasco (Euskal Herriko Unibertsitatra), Avda. Lehendakari Aguirre 83, 48015 Bilbao, España. E-Mail: eupgamom@lg.ehu.es

${ }^{\ddagger}$ Dpto. Economía Aplicada III, misma dirección. E-Mail: az@alcib.bs.ehv.es

${ }_{\S}^{\S}$ Dpto. Economía Aplicada IV, misma dirección. E-Mail: eupcainb@sdx01.bs.ehu.es
} 


\section{Introducción}

El trabajo que presentamos en esta comunicación pretende avanzar en el estudio y desarrollo de las técnicas de elaboración de grafos que sean susceptibles de ser utilizados en combinación con los métodos de Análisis Multivariante Descriptivo de Datos condicionados por una estructura de grafo.

En concreto este artículo propone la metodología para la construcción de dos nuevos grafos a partir de la matriz de datos disponible, la cual va a ser analizada mediante las técnicas citadas. En este caso, aunque el desarrollo que se presenta está pensado para su aplicación a las tablas I-O podría tener un uso mucho más general, basta con que a los datos que se quieran analizar se les asocie unas variables cuya estructura quiera ser controlada en el análisis.

El primero de los grafos que presentamos está basado en la distancia ji-cuadrado por lo que resulta especialmente indicado para un Análisis Factorial de Correspondencias siendo, el segundo, más general al ser apropiado para cualquiera de las técnicas factoriales de análisis de datos condicionadas por una estructura de grafo.

Además de presentar la metodología para la construcción de ambos grafos, el artículo contiene un ejemplo de aplicación de cada uno de ellos.

\section{Grafo intersectorial basado en la distancia ji-cuadrado}

En esta sección vamos a presentar un método para la construcción de un grafo que relaciona a los individuos analizados (sectores o ramas productivas en nuestro caso) con base en los datos proporcionados por variables incluidas en las tablas de Demanda Final o de Inputs Primarios, o por variables exógenas al propio modelo I-O.

La similaridad entre distintos sectores, vértices del grafo, viene asociada a una serie de variables instrumentales que serán objeto de control o estudio en los distintos análisis que se realicen.

Una vez construido el grafo, se presentarán dos algoritmos con los que se buscarán las relaciones maximales de similitud entre sectores. Estas relaciones pondrán de manifiesto agrupaciones de sectores entre los que existe una relación directa que nos ayudarán en la interpretación de los resultados.

Sea $X_{I J}$ la matriz inicial de datos (tabla intermedia de intercambios sectoriales).

Sea $K_{I Q}^{*}$ la matriz $(n \times q)$ en cuyas columnas está representado el conjunto de variables instrumentales, cuya influencia en los resultados del análisis de la tabla $X_{I J}$ se quiere controlar o estudiar (por ejemplo, Tabla de Demanda Final, impuestos, comercio exterior, 
etc.), $Q=\{1, \ldots, j, \ldots, q\}$.

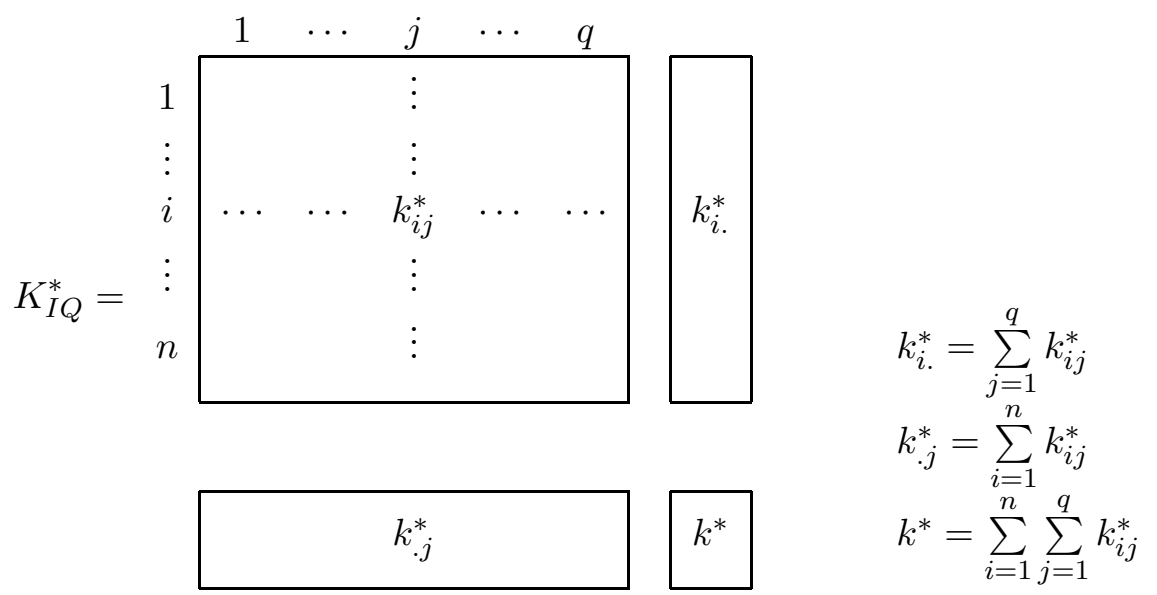

La tabla de frecuencias relativas asociadas a esta matriz se va a expresar de la siguiente forma:

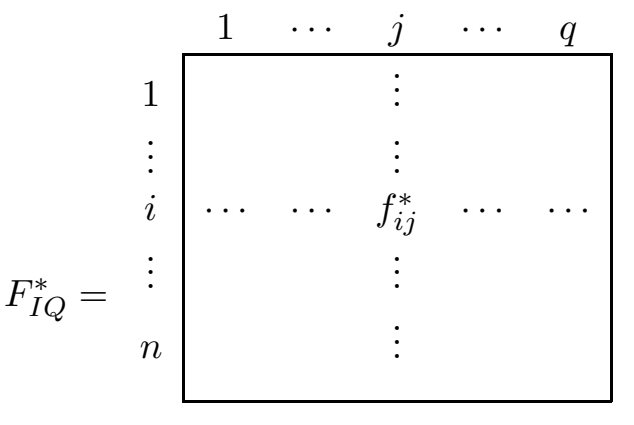

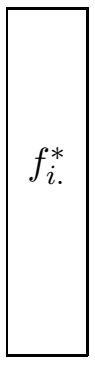

$$
\begin{aligned}
& f_{i j}^{*}=\frac{k_{i j}^{*}}{k^{*}} \\
& f_{i .}^{*}=\sum_{j=1}^{q} f_{i j}^{*}=\frac{k_{i .}^{*}}{k^{*}} \\
& f_{. j}^{*}=\sum_{i=1}^{n} f_{i j}^{*}=\frac{k_{. j}^{*}}{k^{*}} \\
& f_{. j}^{*} \\
& \sum_{i=1}^{n} \sum_{j=1}^{q} f_{i j}^{*}=1
\end{aligned}
$$

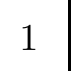

y la de perfiles fila:
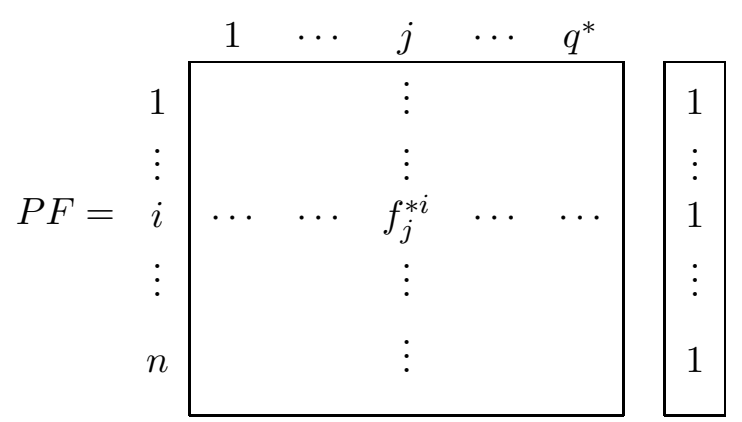

$$
\begin{aligned}
& f_{j}^{* i}=\frac{f_{i j}^{*}}{f_{i .}^{*}} \\
& \sum_{j=1}^{q} f_{j}^{* i}=\sum_{j=1}^{q} \frac{f_{i j}^{*}}{f_{i .}^{*}}=\frac{f_{i .}^{*}}{f_{i .}^{*}}=1
\end{aligned}
$$

donde:

$$
\begin{aligned}
& \text { perfil-fila sector } i: f_{Q}^{* i}=\left\{f_{1}^{* i}, \ldots, f_{j}^{* i}, \ldots, f_{q}^{* i}\right\} \\
& \text { perfil-fila medio } G_{I}:\left\{f_{.1}^{*}, \ldots, f_{. j}^{*}, \ldots, f_{. q}^{*}\right\}
\end{aligned}
$$




\subsection{Obtención del grafo y su matriz asociada}

Una vez construida la tabla de perfiles-fila, se van a calcular las distancias entre ellos para posteriormente definir la similaridad entre sectores en base a dicha distancia. La distancia escogida es la distancia $\chi^{2}$. La elección de esta distancia está justificada por ser la utilizada en el Análisis Factorial de Correspondencias para definir la semejanza entre dos filas o columnas. Entre sus propiedades más importantes destacan, que equilibra la influencia de las columnas sobre la distancia entre las filas debido a la ponderación $\frac{1}{f_{. j}^{*}}$ que da más importancia a los términos más débiles de las modalidades más raras y además cumple el principio de equivalencia distribucional.

La distancia $\chi^{2}$ entre dos perfiles-fila $i$ y $l$ viene definida por:

$$
d_{\chi^{2}}^{2}\left(f_{Q}^{* i}, f_{Q}^{* l}\right)=\sum_{j=1}^{q} \frac{1}{f_{. j}^{*}}\left(\frac{f_{i j}^{*}}{f_{i .}^{*}}-\frac{f_{l j}^{*}}{f_{l .}^{*}}\right)^{2} .
$$

Esta distancia varía entre:

$$
\begin{aligned}
& 0 \leq d_{\chi^{2}}^{2}\left(f_{Q}^{* i}, f_{Q}^{* l}\right) \leq \sum_{j=1}^{q} \frac{1}{f_{. j}^{*}}=C \quad \forall i, l \in I \\
& 0 \leq \sqrt{d_{\chi^{2}}^{2}\left(f_{Q}^{* i}, f_{Q}^{* l}\right)} \leq \sqrt{\sum_{j=1}^{q} \frac{1}{f_{. j}^{*}}}=\sqrt{C} .
\end{aligned}
$$

Con base en todo lo anterior se va a definir la similaridad entre los perfiles de dos individuos $i$ y $l$ (filas de la tabla original) como:

$$
\delta_{i l}=1-\sqrt{\frac{d_{\chi^{2}}^{2}\left(f_{Q}^{* i}, f_{Q}^{* l}\right)}{C}}
$$

y la similaridad entre el perfil del individuo $i$ y el perfil medio:

$$
\delta_{i, G_{I}}=1-\sqrt{\frac{d_{\chi^{2}}^{2}\left(f_{Q}^{* i}, G_{I}\right)}{C}} .
$$

Una vez definida una medida de similaridad entre individuos, sólo queda fijar el criterio por el cual, dos individuos se van a considerar lo suficientemente similares como para estar relacionados por medio de una arista, y así construir el grafo buscado.

Por tanto, fijaremos una cota tal que dos individuos estarán unidos por una arista del grafo si su medida de similaridad es igual o superior a dicha cota. La elección de ésta va a ser determinante en la elaboración del grafo por lo que deberemos ser cuidadosos a la hora de definirla. Proponemos como cotas más interesantes, en función de la matriz de datos utilizada, la similaridad mínima que haya entre cualquier individuo y el perfil medio, la similaridad máxima entre cualquier individuo y el perfil medio o la media de ambas. 
Así, la matriz asociada al grafo, $M(n \times n)$, va a tener de término general (si se escoge la cota mínima):

$$
m_{i j}= \begin{cases}1 & \text { si } \delta_{i j} \geq \min \left\{\delta_{k, G_{I}} ; \forall k=1, \ldots, n\right\} \\ 0 & \text { en cualquier otro caso }\end{cases}
$$

Para la obtención del grafo y de su matriz asociada se han elaborado programas específicos en Fortran.

\subsection{Clasificación de los individuos en base a subrelaciones maximales de similitud}

A partir de las relaciones entre individuos definidas por el grafo anterior, en esta sección se va a proceder a buscar agrupaciones de esos individuos para los que la similaridad $(\delta)$ entre ellos supere en todos los casos la cota marcada anteriormente.

La búsqueda de estas agrupaciones está relacionada con la obtención de subgrafos completos maximales del grafo ordinario asociado. Para su obtención se van a aplicar dos algoritmos: el de Malgrange y el de Pichat.

Antes de describir ambos algoritmos definiremos algunos conceptos básicos para su comprensión. Se denomina submatriz completa de una matriz booleana (0 ó 1) asociada a un grafo a aquella submatriz cuyos elementos son todos unos. Una submatriz principal, también llamada submatriz completa maximal, es una submatriz completa que no está contenida en ninguna otra submatriz completa. Por último, una cobertura de una matriz booleana es un conjunto de submatrices completas que cubren todos los coeficientes de valor 1 de la matriz asociada al grafo.

En general, el problema planteado quedará resuelto cuando se encuentre aquella cobertura cuyo conjunto de submatrices sean, todas ellas, submatrices principales o completas maximales. De éstas, aquéllas que sean cuadradas determinarán las subrelaciones de similitud buscadas que definen a aquellos individuos pertenecientes a una misma agrupación.

\subsubsection{Algoritmo de Malgrange para la obtención de submatrices completas maximales [5]}

Sea $M$ la matriz asociada a un grafo $G$, siendo $I$ el conjunto de las filas y $J$ el conjunto de las columnas. Cada submatriz completa va a estar definida por la pareja de subconjuntos ordinarios $\left(I_{p}, J_{q}\right)$ tal que $I_{p} \subset I$ y $J_{q} \subset J$.

Se demuestra que las operaciones $\dot{\cup}$ y $\dot{\cap}$ que hacen corresponder a dos submatrices completas cualesquiera de $M$ :

$$
\begin{gathered}
M_{k} \text { definida por }\left(I_{k}, J_{k}\right) \\
M_{l} \text { definida por }\left(I_{l}, J_{l}\right)
\end{gathered}
$$

las dos submatrices $M^{\prime}$ y $M^{\prime \prime}$

$$
\begin{aligned}
& M_{k} \dot{\cup} M_{l}=M^{\prime} \text { definida por }\left(I_{k} \cup I_{l}, J_{k} \cap J_{l}\right) \\
& M_{k} \dot{\cap} M_{l}=M^{\prime \prime} \text { definida por }\left(I_{k} \cap I_{l}, J_{k} \cup J_{l}\right)
\end{aligned}
$$


son operaciones internas en el conjunto de submatrices completas de $M$.

Para obtener todas las submatrices principales de $M$ en un número finito de pasos, bastará con aplicar a las submatrices completas de una cobertura $C$ las dos reglas siguientes de forma alternativa, hasta que ya no sea posible encontrar submatrices completas que, o no sean iguales o no estén contenidas en las obtenidas en el paso precedente.

Sea la cobertura $C=\left\{M_{1}, M_{2}, \ldots, M_{p}\right\}$. Las dos reglas citadas son:

- Primera regla. Suprimir toda matriz $M_{k}$ contenida en otra matriz $M_{l}$ de la cobertura $C$.

- Segunda regla. Añadir a $C$ las submatrices obtenidas por las operaciones $\dot{U}$ y $\dot{n}$ definidas anteriormente y aplicadas a todos los pares de matrices $M_{k}$ y $M_{l}$ conservadas en la cobertura (excepto si la submatriz completa está contenida en una submatriz que figure en $C$, lo que evita un proceso infinito).

Por último, como ya se dijo anteriormente, de esas submatrices principales que forman una cobertura de la matriz booleana $M$ asociada al grafo, aquellas que sean cuadradas darán las subrelaciones de similitud buscadas. Estas matrices cuadradas cruzarán los individuos cuya similaridad entre sus perfiles supere la cota marcada para determinar el grafo.

Una recomendación práctica para que la cobertura inicial de submatrices completas sea bastante cercana a la que se busca es, comenzar reordenando la matriz $M$ de tal forma que se obtengan submatrices completas cuadradas. Para ello, se colocan las columnas de la matriz en orden al mayor número de unos.

\subsubsection{Algoritmo de Pichat [5]}

Este algoritmo es más rápido y sencillo que el de Malgrange, sin embargo, sólo es válido para matrices booleanas cuadradas y simétricas con lo que aquél es más general que éste.

El método se desarrolla de la siguiente forma. Considerando sólo la semimatriz que está por encima de la diagonal principal, para cada cero de las filas de dicha matriz (tomando los elementos como variables booleanas) se asocia mediante el signo suma booleana $(\dot{+})$ el individuo correspondiente a la fila tomada y los individuos de aquellas columnas donde se encuentren los ceros, a los que se les aplicará el producto booleano $(\cdot)^{1}$. Si no hay ceros en la fila se considerará que la suma es igual a 1.

Posteriormente, se hace el producto de los resultados de cada fila y se expresa la función obtenida en términos minimales, es decir, simplificando las expresiones booleanas en base a las siguientes propiedades:

- $x \dot{+} x=x$

- $x \cdot x=x$

\footnotetext{
${ }^{1}$ Estas potencias son obtenidas por cálculo booleano, donde las operaciones suma $(\dot{+})$ y producto $(\cdot)$ cumplen las propiedades:

$1 \dot{+} 1=0 \dot{+} 1=1 \dot{+} 0=1 ; 0 \dot{+} 0=0$

$1 \cdot 1=1 ; 1 \cdot 0=0 \cdot 1=0 \cdot 0=0$
} 


$$
\text { - } x \dot{+} x y=x
$$

Una vez obtenida esta expresión a la que genéricamente se le denominará $S$, se toman los complementarios de cada término que describirán las subrelaciones maximales que constituyen la cobertura.

\section{$2.3 \quad$ Ejemplo}

El conjunto de individuos está formado por los 38 sectores o ramas de actividad de las Tablas I-O de la Comunidad Autónoma del País Vasco. Las variables instrumentales son las exportaciones de cada uno de ellos al resto del estado, a los países de la C.E.E. y al resto del mundo. En este caso la cota utilizada es la semejanza media que haya entre cualquier individuo y el perfil-fila medio. Luego, dos individuos estarán unidos mediante una arista si su similaridad es igual o mayor que esa similaridad media. La matriz asociada al grafo $M$ tiene por término general:

$$
m_{i j}= \begin{cases}1 & \text { si } \delta_{i j} \geq \frac{1}{n} \sum_{i=1}^{n} \delta_{i, G_{I}} \\ 0 & \text { en cualquier otro caso }\end{cases}
$$

siendo $n=38$ el número de sectores, $\delta_{i j}$ la similaridad entre los perfiles de los individuos $i$ y $j$ y $\delta_{i, G_{I}}$ la semejanza entre el perfil del individuo $i$ y el perfil medio.

La matriz de incidencia, con una cota de 0,908, se muestra en la Tabla 1.

De la mera observación de esta matriz se pueden destacar algunas características de las uniones resultantes:

- Los sectores Construcción (s.09), Transporte de viajeros por carretera (s.24), Transporte ferroviario (s.26), Comunicaciones (s.29), Alquiler de inmuebles (s.33), Sanidad comercial (s.34), Enseñanza comercial (s.35), Servicios personales y recreativos (s.36), Administraciones públicas (s.37) y Otros servicios (s.38) forman una clase en la que todos ellos están unidos entre sí. Esto de debe a que sus exportaciones, tanto al resto del estado, a los países de la C.E.E. y al resto del mundo, son nulas.

- Además, hay tres vértices en este grafo que se encuentran aislados y que se corresponden con las ramas Caucho y neumáticos (s.12), Transporte marítimo y aéreo (s.27) y Bancos (s.30). En los tres casos el perfil exportador que presentan es claramente diferente a los del resto lo que hace precisamente que no estén asociados a ningún otro sector. Mientras que el primero de ellos tiene unos niveles similares en los tres tipos de exportaciones, el Transporte marítimo y aéreo destaca por sus altas exportaciones al resto del mundo y el bajo nivel en las que van a parar al resto del estado y el sector de Bancos es el de mayores exportaciones a los países de la C.E.E., de los 38 estudiados, con un porcentaje muy por debajo de la media en las que son destinadas al resto del mundo y algo por debajo de la media en las del resto del estado.

- Del resto de relaciones que aparecen en el grafo se hace realmente difícil el poder extraer conclusiones sin realizar otro tipo de análisis. Sin embargo, sí se pueden 


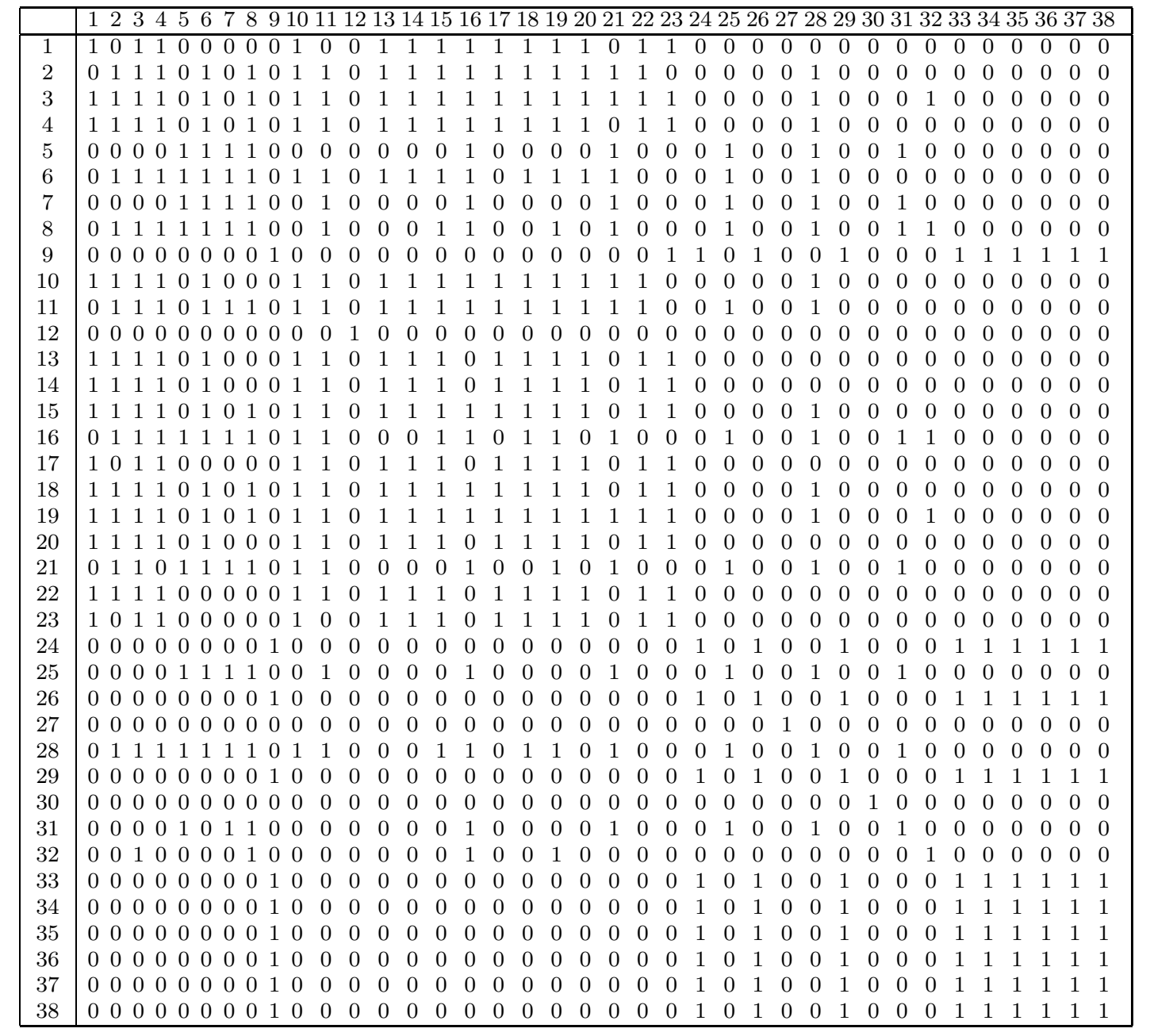

Tabla 1: Matriz de incidencia con una cota de 0,908.

destacar una serie de sectores con asociaciones similares en los que las exportaciones al resto del estado están por debajo de la media mientras que las destinadas al extranjero (tanto a países de la C.E.E. como al resto del mundo) están por encima. Estos sectores son: Metálicas básicas (s.05), Metálicas finales (s.07), Textil (s.16), Comercio (s.21), Transporte de mercancías por carretera (s.25) y Seguros (s.31).

Una vez obtenido este grafo se podría realizar un Análisis de Correspondencias de la tabla intermedia de inputs expuesta anteriormente, en el que el estudio de las relaciones intersectoriales estaría condicionado por las asociaciones que se den entre sectores dependiendo de su perfil exportador.

Además, y de forma complementaria, también se podría elaborar un grafo de partición a partir del anterior, con el que clasificar las distintas ramas dependiendo de su volumen de exportaciones y del destino de las mismas, para después analizar la tabla de datos con los análisis alisado y de diferencias locales. Esta partición se podría obtener con alguna de 
las técnicas de clasificación conocidas como la que se consigue a partir de la construcción del árbol de expansión minimal o árbol parcial mínimo. En este caso, el árbol sólo se elaboraría para los sectores no incluidos en los dos primeros apartados anteriores, ya que tanto los del primer punto como los sectores aislados constituirían clases del grafo.

\section{Grafo de partición basado en las componentes fuerte- mente conectadas}

El grafo que vamos a presentar a continuación tiene, al igual que el anterior, un ámbito de aplicación mucho más general que el de las tablas I-O.

La partición del conjunto de sectores que se va a realizar en este apartado, está basada en el concepto de componentes fuertemente conectadas de la teoría de grafos. La idea intuitiva de este método es que dos vértices de un grafo pertenecerán a la misma clase si entre ellos existe una relación directa o indirecta con base en relaciones de transitividad. Por tanto, el punto de partida es contar con una estructura de grafo.

Sea un grafo dirigido y reflexivo como, por ejemplo, el que podemos construir a partir de la matriz de coeficientes técnicos (donde los vértices son los sectores), en el que existirá el arco $(i, j)$ si el coeficiente $a_{i j}(\forall i, j=1, \ldots, n)$ supera una cierta cota determinada a priori. La matriz booleana asociada a dicho grafo es $M(n \times n)$.

Se dice que dos vértices $i, j$ están fuertemente conectados si existe al menos un camino que une el vértice $i$ con el $j$ y un camino que une $j$ con $i$. Esta relación de fuerte conexión va a determinar una partición de los vértices del grafo en clases de equivalencia a las que llamaremos componentes fuertemente conectadas. La unión de los vértices de cada una de las clases de equivalencia dará como resultado un grafo de partición. Es posible que el resultado sea un grafo con un número de vértices demasiado elevado como para poder ser utilizado, en este caso se llega a obtener el grafo de partición en base a criterios subjetivos en función del análisis de los resultados obtenidos.

La potencia $M^{s}$, obtenida por cálculo booleano nos informa sobre los caminos existentes de longitud $s$ :

$$
m_{i j}^{s}= \begin{cases}1 & \text { si existe un camino que une } i \text { con } j \\ 0 & \text { si no existe un camino que une } i \text { con } j\end{cases}
$$

Si se calcula la sucesión de potencias de la matriz de $\operatorname{arcos} M, M^{2}, \ldots$, se denomina cerradura transitiva [4]:

$$
\hat{M}=M \dot{+} M^{2} \dot{+} \ldots \dot{+} M^{s} \dot{+} \ldots
$$

Para un grafo de orden $r$, todo camino elemental es de longitud menor o igual a $r-1$. De aquí, que la matriz $M \dot{+} M^{2} \dot{+} \ldots \dot{+} M^{(r-1)}$, proporciona todos los caminos cuya longitud es menor o igual que $r-1$. A partir de esta potencia, la suma booleana de dichas matrices proporciona los mismos caminos. Por tanto, la cerradura transitiva se puede calcular como:

$$
\hat{M}=M \dot{+} M^{2} \dot{+} \ldots \dot{+} M^{(r-1)}
$$

que determina las componentes fuertemente conectadas del grafo y, por tanto, una partición de los vértices del mismo. 


\subsection{Ejemplo}

Para llegar a una estructura de partición, con base en la idea de que dos individuos pertenecen a la misma clase si entre ellos existe una relación directa o indirecta a través de relaciones de transitividad, necesitamos partir de un grafo dirigido y reflexivo, el cual va a ser elaborado a partir de la matriz intermedia de intercambios correspondiente a los 38 sectores ya citados.

El criterio seguido para la construcción de dicho grafo es el siguiente: consideramos que el sector $j$ depende del sector $i$ si la demanda relativa de inputs del sector $i$ por parte del sector $j$ (con respecto al valor de la producción del sector $j$ ) es superior a una determinada cota, prefijada de antemano. En este caso la matriz de incidencia asociada al grafo tiene por término general:

$$
m_{i j}= \begin{cases}1 & \text { si existe } \frac{x_{i j}}{X_{j}} \geq \operatorname{cota} \\ 0 & \text { si } \frac{x_{i j}}{X_{j}}<\operatorname{cota}\end{cases}
$$

siendo:

$x_{i j}$ la cantidad de input del sector $j$ procedente del sector $i$.

$X_{j}$ el valor de la Producción Efectiva del sector $j$.

La cota utilizada en este caso para la construcción del grafo ha sido de 0,025, es decir que un sector dependerá de otro si para su producción demanda al menos un $2,5 \%$ de inputs de éste. La elección de esta cota es completamente arbitraria y, en nuestro caso, se ha tomado la decisión en función de un sencillo análisis descriptivo previo de los datos. La matriz de incidencia resultante se muestra en la Tabla 2.

Observando una fila cualquiera de esta matriz (fila $i$-ésima), tenemos los sectores que dependen directamente (al nivel prefijado) del sector $i$. Serán aquellos en los que aparezca un 1 en la casilla correspondiente.

Una vez que se ha obtenido el grafo correspondiente calculamos la cerradura transitiva que determina las componentes fuertemente conectadas. En la Tabla 3 se muestra el resultado, en el que se han ordenado las filas de mayor a menor número de unos, de tal forma que su estudio se haga más sencillo.

Del análisis de esta matriz podemos destacar las siguientes conclusiones:

- Existe una componente fuertemente conectada formada por 16 sectores, a la que vamos a denominar G1, en la cada uno de ellos depende de los demás. Estos sectores son:

- Sectores agrarios (s.01) - Papel y art. gráficas (s.15)

- Ind. agroalimentarias (s.03) - Recuperación y rep. (s.20)

- Energía (s.04) - Comercio (s.21)

- Met. básicas (s.05)

- Trans. merc. carretera (s.25)

- Met. intermedias (s.06) - Anexos al transporte (s.28)

- Met. finales (s.07)

- Serv. a empresas (s.32)

- Ind. no metálicas (s.08)

- Alquiler de inm. (s.33)

- Construcción (s.09)

- Servc. personales (s.36) 
Tabla 2: Matriz de incidencia con una cota de 0,025.

Este conjunto de ramas productivas son las más fuertes (excepto Comunicaciones (s.29)) desde el punto de vista de la dependencia ya que la mayoría de los sectores restantes dependen de ellas y, a su vez, ellas no dependen de ninguna otra excepto de Comunicaciones.

Por tanto, tenemos un sector aislado, el de las Comunicaciones (s.29), que se destaca de todos los demás por ser aquel que más sectores dependen de él sin depender él de nadie.

- Siguiendo con el análisis de la matriz, nos encontramos con un grupo de sectores que, aún sin ser una componente fuertemente conectada, tienen características similares. Cada uno de ellos tiene independencia absoluta respecto al resto (incluidos los demás del grupo) ya que no dependen de nadie pero tampoco los demás dependen de ellos. Este grupo lo forman: Textil (s.16), Confección (s.17), Cuero y calzado (s.18). Además hemos añadido el sector Sanidad comercial (s.34) ya que no depende de 
2936333212825212015987654334241411222321626271718303119121335103738

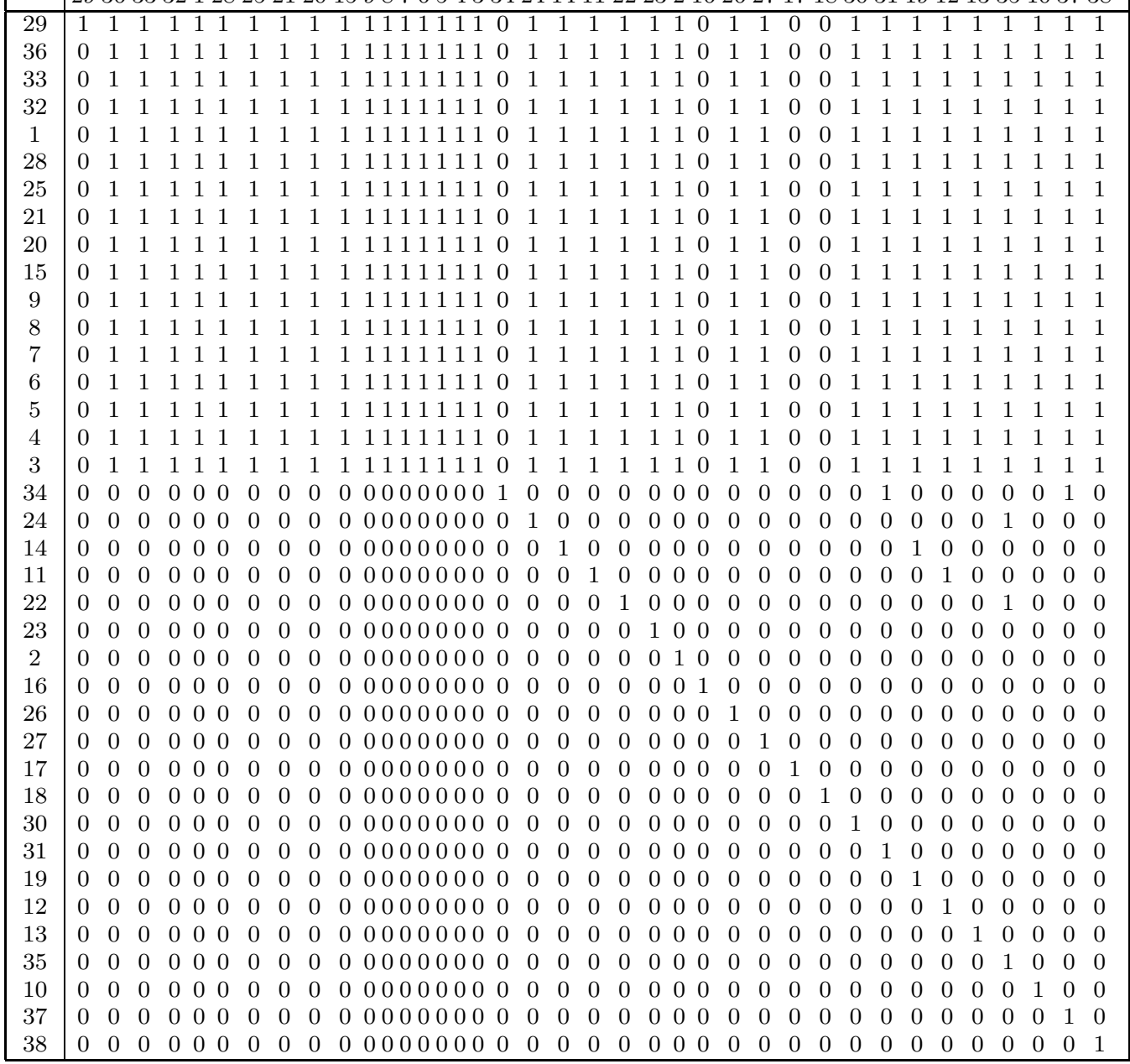

Tabla 3: Matriz resultante al calcular la cerradura transitiva que determina las componentes fuertemente conectadas.

nadie y él sólo depende de los sectores Seguros (s.31) y Administraciones públicas (s.37).

- Por último, tenemos otro conjunto cuyas características comunes son que pocos sectores dependen de ellos aunque, ellos sí que dependen directamente del grupo comentado en el primer apartado $(\mathbf{G 1})$. Esta agrupación, que tampoco forma una componente fuertemente conectada, está formada por 17 sectores que los vamos a clasificar en dos subgrupos:

- aquéllos con algún sector dependiente de ellos 
- Química (s.11)

- Madera (s.14)

- Hostelería (s.22)

- Transporte de viajeros por carretera (s.24)

- aquéllos de los que no depende ningún sector

- Pesca (s.02)

- Vidrio (s.10)

- Caucho y neumáticos (s.12)

- Plástico (s.13)

- Otras manufacturas (s.19)

- Transporte urbano (s.23)

- Transporte ferroviario (s.26)

- Transporte marítimo y aereo (s.27)

- Bancos (s.30)

- Seguros (s.31)

- Enseñanza comercial (s.35)

- Administraciones públicas (s.37)

- Otros servicios (s.38)

Dado que la componente fuertemente conectada G1 está compuesta por un número muy elevado de sectores, nos hemos decidido por realizar un segundo paso que consiste en repetir el análisis de un grafo reducido en el que uno de sus nodos es la citada componente. En esta ocasión la cota utilizada ha sido de un $3 \%$ con el fin de matizar más las relaciones de dependencia. El estudio de estos resultados nos llevan a distinguir tres niveles, respecto a la fortaleza de los sectores, en relación a su mayor o menor nivel de dependencia. Estos tres niveles son:

- En el nivel 1 (N1) tenemos los sectores Energía (s.4) y Servicios personales (s.36) para los cuales, el resto de sectores de G1 dependen de ellos y ellos no dependen de nadie excepto, lógicamente, de Comunicaciones (s.29). Ambas ramas, por separado, forman una componente fuertemente conectada.

- El segundo nivel (N2) está compuesto por Papel y artes gráficas (s.15), Comercio (s.21) y Servicios a empresas (s.32). Estos tres sectores forman una componente fuertemente conectada y dependen del N1 y de ningún otro.

- El resto de sectores de G1 se encuentran en el nivel 3 (N3). Entre todos ellos tenemos que distinguir:

- Una componente fuertemente conectada compuesta por las ramas metálicas: Metálicas básicas (s.05), Metálicas intermedias (s.06) y Metálicas finales (s.07), además de Recuperación y reparación (s.20).

- Los sectores Industrias agroalimentarias (s.03) y Sectores agrarios (s.01) los cuales forman otra componente fuertemente conectada. 
- El resto de sectores. Industrias no metálicas (s.08), Transporte de mercancías por carretera (s.25), Anexos al transporte (s.28), Construcción (s.09) y Alquiler de inmuebles (s.33). Los tres primeros, aún sin ser una componente fuertemente conectada, tienen muchas relaciones de dependencia entre ellos. Los dos últimos son atípicos ya que, de pertenecer al grupo de los más fuertes (en cuanto a que los demás dependen de ellos) con una cota del 2,5\%, pasan a que ningún sector depende del s.33 y solamente los sectores 22, 26, 33 y 35 dependen del s.09 con una cota del 3\%. Esto nos lleva a pensar que son ramas muy homogéneas respecto a la proporción estudiada y cuyos valores oscilan entre estas dos cantidades.

Cada uno de los sectores mencionados en este punto formarían, por sí mismos, una componente fuertemente conectada y dependen de los dos niveles anteriores.

En el escmema. (Gráfico 1) nodemos ver cómo se han nerfilado lns comnortamientos de los sector

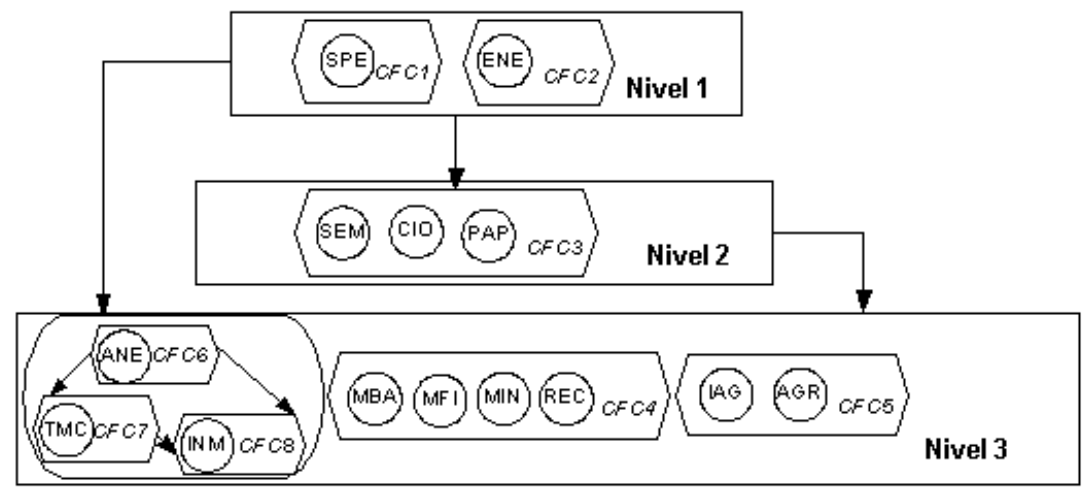

Gráfico 1: Perfil de comportamientos de los sectores del grupo G1.

En base a los resultados que hemos expuesto y, por tanto, siguiendo criterios subjetivos llegamos a la siguiente estructura de partición susceptible de ser analizada con las técnicas propuestas en este trabajo:

- CLASE 1 (C1): $\left\{\begin{array}{l}\text { Comunicaciones (s.29) } \\ \text { Energía (s.04) } \\ \text { Servicios personales (s.36) }\end{array}\right.$

- CLASE 2 (C2): $\left\{\begin{array}{l}\text { Papel y artes gráficas (s.15) } \\ \text { Comercio (s.21) } \\ \text { Servicios a empresas (s.32) }\end{array}\right.$

- CLASE 3 (C3): $\left\{\begin{array}{l}\text { Metálicas básicas (s.05) } \\ \text { Metálicas intermedias (s.06) } \\ \text { Metálicas finales (s.07) } \\ \text { Recuperación y reparación (s.20) }\end{array}\right.$ 
- CLASE $4(\mathbf{C 4}):\left\{\begin{array}{l}\text { Sectores agrarios (s.01) } \\ \text { Industrias agroalimentarias (s.03) }\end{array}\right.$

- CLASE $5(\mathbf{C 5}):\left\{\begin{array}{l}\text { Industrias no metálicas (s.08) } \\ \text { Transporte de mercancías por carretera (s.25) } \\ \text { Anexos al transporte (s.28) } \\ \text { Construcción (s.09) } \\ \text { Alquiler de inmuebles (s.33) }\end{array}\right.$

- CLASE 6 (C6): $\left\{\begin{array}{l}\text { - Química (s.11) } \\ \text { - Madera (s.14) } \\ \text { - Hostelería (s.22) } \\ \text { - Transporte de viajeros por carretera (s.24) }\end{array}\right.$

- CLASE 7 (C7): $\left\{\begin{array}{l}\text { - Pesca (s.02) } \\ \text { - Vidrio (s.10) } \\ \text { - Caucho y neumáticos (s.12) } \\ \text { - Plástico (s.13) } \\ \text { - Otras manufacturas (s.19) } \\ \text { - Transporte urbano (s.23) } \\ \text { - Transporte ferroviario (s.26) } \\ \text { - Bancos (s.30) } \\ \text { - Seguros (s.31) } \\ \text { - Enseñanza comercial (s.35) } \\ \text { - Administraciones públicas (s.37) } \\ \text { - Otros servicios (s.38) }\end{array}\right.$

- CLASE 8 (C8): $\left\{\begin{array}{l}\text { Textil } \text { (s.16) } \\ \text { Confección (s.17) } \\ \text { Cuero y calzado (s.18) } \\ \text { Sanidad comercial (s.34) }\end{array}\right.$

\section{Conclusiones}

La primera, y la mas importante, de las conclusiones que podemos obtener de este trabajo es que ambos grafos resultan adecuados para ser utilizados en las distintas técnicas factoriales descriptivas del análisis multivariante de datos, por lo tanto, son un complemento de gran importancia en las distintas metodologías descritas en la literatura existente.

El grafo basado en la distancia ji-cuadrado resulta especialmente adecuado cuando la técnica a emplear en el trabajo que estemos realizando sea un Análisis de Correspondencias, mientras que el basado en las componentes fuertemente conectadas será utilizado si lo que se desea es conseguir una partición del conjunto de individuos teniendo en cuenta las relaciones tanto directas como indirectas entre ellos. 
Por último, cabe destacar, como se puede ver a través de los ejemplos, la validez y la oportunidad de ambos grafos en análisis de las economías de los países o regiones basados en las Tablas Input-Output.

\section{Referencias}

[1] Aluja, T.: Lebart, L. (1985) "Factorial analysis upon a graph", Bulletin Technique du CESIA 3: 4-34.

[2] Benali, H.; Escofier, B. (1990) "Analyse factorielle lissée et analyse factorielle des différences locales", Rev. Statistique Appliquée 38(2): 55-76.

[3] García Montoya, M.A. (1998) Tratamiento Factorial de Estructuras definidas mediante Grafos. Aplicación al Estudio de las Tablas Input-Output. Tesis Doctoral, U.P.V./E.H.U., Bilbao.

[4] Kaufmann, A. (1971) Métodos y Modelos de la Investigación de Operaciones, $1^{a}$ Ed. Continental, México.

[5] Kaufmann, A. (1982) Introducción a la Teoría de los Conjuntos Borrosos, volumen I. Continente, Mexico.

[6] Martínez Arnaiz, J.A. (1987) Aplicación del Análisis Multivariante y de la Teoría de Grafos al Estudio de Ciertas Cuestiones Sociodemográficas en España. Tesis doctoral, FCEE, Universidad del País Vasco/E.H.U., Bilbao. 